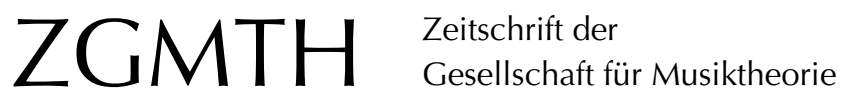

Krämer, Laura (2012): Form und Soziolekt in Schuberts Tänzen. ZGMTH 9/1, 69-80. https://doi.org/10.31751/659

(C) 2012 Laura Krämer

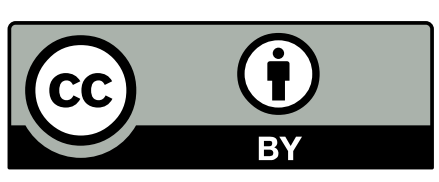

Dieser Text erscheint im Open Access und ist lizenziert unter einer Creative Commons Namensnennung 4.0 International Lizenz.

This is an open access article licensed under a

Creative Commons Attribution 4.0 International License.

veröffentlicht / first published: 26/11/2012

zuletzt geändert / last updated: 02/07/2013 


\title{
Form und Soziolekt in Schuberts Tänzen
}

\author{
Laura Krämer
}

ABSTRACT: In Schuberts Tänzen für Klavier verbinden sich Gestaltungsweisen der alpenländischen Volksmusik und der Kunstmusik des 18. und 19. Jahrhunderts. Zwar verlieren die Bezeichnungen 'Ländlerı, 'Deutscher Tanz und 'Walzer in der Kunstmusik weitgehend ihre differenzierende Funktion, doch verweisen Idiomatik und Formbildung bei Schubert auf die sozialen Sphären, denen die verschiedenen volksmusikalischen Tanzsatztypen jeweils angehörten: Schuberts Tänze schweben gleichsam zwischen verschiedenen musikalischen Soziolekten. Zugleich markieren sie eine Wegscheide: Führt die Adaption ternärer Tänze der Ländler- bzw. Walzergattung bei Strauß und Sohn zum symphonischen Walzer mit Introduktion und Coda, so bereiten Schuberts Tänze dem lyrischen Klavierstück den Weg.

Die Eigenschaften von Musik können, ähnlich wie die einer natürlichen Sprache, auf eine bestimmte Zeit (diachron), Region (dialektal) oder soziale Schicht (soziolektal) hinweisen. In der soziolektal geprägten Sprachsituation werden die einer bestimmten sozialen Sphäre angehörenden Momente als selbstverständlich vorausgesetzt, in der Kunstmusik bisweilen auch gezielt aufgerufen. Die intentionale Inanspruchnahme von Soziolekten kann im Sinne einer Zeichenrelation als Topos $^{1}$ oder indexikalischer Verweis ${ }^{2}$ gedeutet werden.

Tänze gehören zu den am stärksten sozial konnotierten Musikgattungen. Die für einen bestimmten Tanz einschlägigen musikalischen Gestaltungsweisen sind daher geeignet, auf ihre soziale Herkunftsebene zu verweisen. Gerade Schuberts Tänze für Klavier geben zahlreiche Beispiele für den Gebrauch und das Ineinandergreifen verschiedener musikalischer Soziolekte. Sie bewegen sich im Spannungsfeld zwischen expliziten Tanz-

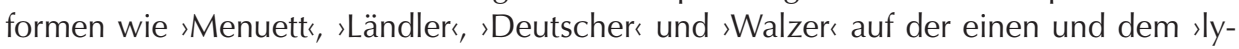
rischen Klavierstückı auf der anderen Seite. Diese Gattungen sind jeweils unterschiedlichen sozialen Schichten zugeordnet: das Menuett dem Adel, Ländler, Deutscher und auch der Walzer, bevor er das Menuett bei Hofe verdrängte, der einfachen Land- und Stadtbevölkerung, und das lyrische Klavierstück dem ins Private zurückgezogenen bürgerlichen Individuum. Zeitlich repräsentiert das Menuett die Hochkultur der Vergangen-

1 Als Topoi bezeichnet Josef Gmeiner (1979) kodierte soziologische Merkmale von Musik. Nach dem Vorbild Gmeiners wird in dieser Arbeit generell der Begriff des musikalischen Soziolekts gebraucht.

2 Die Begriffsverwendung orientiert sich an der musikalischen Semantik Vladimir Karbusickys (1986). Beim indexikalischen Verweis wird durch Aufgreifen einer analogen Gestaltungsweise auf einen anderen Stil verwiesen, dessen Assoziationspotential dadurch für die verweisende Musik aktiviert wird. 
heit, während das lyrische Klavierstück in die Zukunft einer neuen, bürgerlichen Gesellschaft weist. Die Volksmusik schließlich ist zwar nicht tatsächlich, aber in dem, was sie symbolisiert, zeitloses und snaturwüchsiges` Allgemeingut. Da Belange des Soziolektes immer auch solche der Stilhöhe berühren, verwundert es nicht, dass Schuberts Tänze in der musikwissenschaftlichen Literatur der letzten Jahrzehnte primär hinsichtlich der Frage diskutiert wurden, inwieweit sie als (hohe) Kunst- oder (triviale) Gebrauchsmusik zu betrachten seien. ${ }^{3}$

Gegenstand der folgenden Betrachtung ist die musiksoziologische Aussagekraft der musikalischen Form. Anhand von Phrasenbildung, Periodik, Gesamtform und harmonischer Disposition sowie der Gruppierung der Stücke soll in Schuberts Tänzen das Verschmelzen verschiedener Stilebenen zu einem sschwebenden Soziolekt s gezeigt werden. Auch andere, ebenfalls stark soziolektal konnotierte Ebenen der Komposition, etwa Melodik und Begleittextur, werden berücksichtigt.

Eine Begriffsklärung wird dadurch erschwert, dass die Bezeichnungen >Deutscher Tanzı, 'Ländler und 'Walzer zwar choreographische und (mit geringerer Trennschärfe) auch musikalische Differenzen markieren, ihr Gebrauch zur Schubert-Zeit jedoch keineswegs eindeutig ist. ${ }^{4}$

Das Charakteristikum des `Deutschen Tanzes` ist das `Walzen`: Die Paare umfassen sich mit einem Arm, der freie Arm wird ausgestreckt und die beiden freien Hände ineinandergelegt. Die einzelnen Paare bilden einen großen Kreis und umrunden in drehender Bewegung den Saal. Im Inneren des Kreises versuchen sich einzelne Paare gegebenenfalls an diversen anderen Figuren.

Beim `Walzer hingegen wird ausschließlich gewalzt. In seiner arrivierten Form unterscheidet er sich von den `Deutschen und `Ländlern ‘ durch die 16- statt 8-Taktigkeit der formalen Einheiten, das höhere Tempo sowie durch die Zusammenfassung einer Kette von Walzern durch Introduktion und Coda ('Walzer heißt demnach sowohl der einzelne Tanzsatz als auch die Reihungsform im Ganzen). Allerdings wurde die Bezeichnung ,Walzer von Schuberts Verlegern auch für die viel kleineren, auf achttaktiger Periodik beruhenden Formen seiner Tänze gebraucht; in Schuberts Autographen jedoch findet sie sich kein einziges Mal. ${ }^{5}$ In den folgenden Ausführungen wird daher auf den Begriff des ,Walzers` verzichtet.

3 Vgl. Hilmar 1985, 76ff., Kap. „Der überschätzte Tanzkomponist», sowie Mahlert 2005, insbes. $169 \mathrm{ff}$.

4 So konstatiert Walburga Litschauer im Vorwort zur Ausgabe der Tänze (Bd. 1) im Rahmen der NGA, X, Sp. 3: „Die Abgrenzung vom Deutschen zum Ländler ist bei Schubert - wie überhaupt im frühen 19. Jahrhundert - nicht konsequent durchführbar, da derselbe Tanz gelegentlich unter verschiedenen Bezeichnungen in mehrere Tanzmanuskripten aufscheinen kann. Dies ist etwa bei D 365/2 und 3, sowie bei D 366/4 der Fall. Die genannten Tänze hat Schubert selbst einmal als ,Ländler und einmal als >Deutscher bezeichnet."

5 Im Vorwort der NGA zur Ausgabe der Tänze (Bd. 1) heißt es: „Wie schon erwähnt, hat Schubert selbst in seinen Autographen nur einmal einen Tanz als Walzer bezeichnet.« (XI, Sp. 2). Nicht nur aber ist diese Erwähnung im Vorhergehenden nicht auffindbar, zudem gehört der gemeinte Tanz D 365/3, wie demselben Band zu entnehmen ist, einem Manuskript an, das Schubert mit »Deutsche« überschrieben hat. 


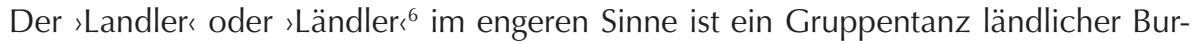
schenverbände. Im städtischen Milieu dagegen dient der Begriff >Ländler` zur SchubertZeit unspezifisch als Sammelbezeichnung für Tänze im Dreivierteltakt mit 〉ländlichem Einschlag ‘ ${ }^{7}$, so dass die Begriffe 'Ländler`, 'Deutscher (und mit Einschränkung auch 'Walzerı) mehr oder weniger zusammenfallen. ${ }^{8}$ Im Folgenden möchte ich daran anknüpfend das Volksmusikalische als das 'Ländlerischer bezeichnen.

Im Folgenden werden zunächst anhand von Ländlern und Menuetten Schuberts die formalen Merkmale des Volkstümlichen und des Traditionell-Kunstmusikalischen einander gegenübergestellt. ${ }^{9}$ In einem zweiten Schritt wird gezeigt, inwiefern musikalische Soziolekte in Schuberts Formbildung ineinanderfließen. Abschließend wird versucht, eine Entwicklungslinie von Schuberts Ländlern zum lyrischen Klavierstück aufzuzeigen.

\section{Volkstümlich-ländlerische Formbildung}

Folgende Merkmale kennzeichnen den volkstümlichen Ländler:

- Ein Ländler umfasst 16 Takte.

- Mehrere Ländler meist derselben Tonart gruppieren sich zu einer Kette, die jedoch bei Schubert keine formale Rahmung durch eine Introduktion und/oder Coda erhält. ${ }^{10}$

- Ein 16-taktiger Ländler besteht aus zwei jeweils wiederholten Achttaktern ohne Erweiterungen.

- Die Achttakter sind jeweils als nichtmodulierende Perioden angelegt, jedoch mit imperfektem Ganzschluss statt Halbschluss in der Mitte. Die Periode mit imperfektem Ganzschluss ist die einfachere Formbildung gegenüber derjenigen mit Halbschluss, da die analoge Bildung einer Schlusskadenz im zweiten Viertakter geringerer Veränderungen des musikalischen Materials bedarf.

- Der zweite Achttakter ist in motivischer Hinsicht entweder nicht auf den ersten Achttakter bezogen (zweiteilige Liedform), oder aber er ergibt sich aus typisch volkstümlichen Verfahrensweisen der Taktumstellung bzw. Wiederverwendung einzelner Takte, insbesondere der Schlussformeln. Untypisch ist jede Form von >dialektischer Formbildung (wie z. B. in der dreiteiligen Liedform).

6 Die Schreibweise >Ländler geht wahrscheinlich auf die Umschrift des hellen mundartlichen sa im Ursprungswort >Landler` zurück. Vgl. Derschmidt/Deutsch 1998, 18.

7 Das ländliche Pendant zum städtischen Paartanz war eher der 'Steyerische Tanzı, der auch in musikalischer Hinsicht mehr als Vorgänger des Walzers gelten darf als der Landler.

8 Auch im Rahmen der gegenwärtigen österreichischen Ethnomusikologie wird die Bezeichnung Landler bzw. Ländler als Oberbegriff für alle ternären Tänze benutzt.

9 Der besseren Vergleichbarkeit halber ziehe ich von Schubert nur 16-taktige Tänze heran (nur wenige Tänze weisen andere Taktanzahlen auf).

10 Carl Maria von Weber, Joseph Lanner und vor allem Vater und Sohn Strauß bildeten aus Walzerketten durch motivisch und formal verklammernde Introduktionen und Codas eine neue Großform und rückten so den Walzer von der Gebrauchsmusik in Richtung Hochkultur. Bei Schubert gibt es zu diesem Verfahren nur vereinzelte Ansätze (vgl. die »Introduzione« zu D 128), in den zu Lebzeiten veröffentlichten Sammlungen spielt es keine Rolle. 

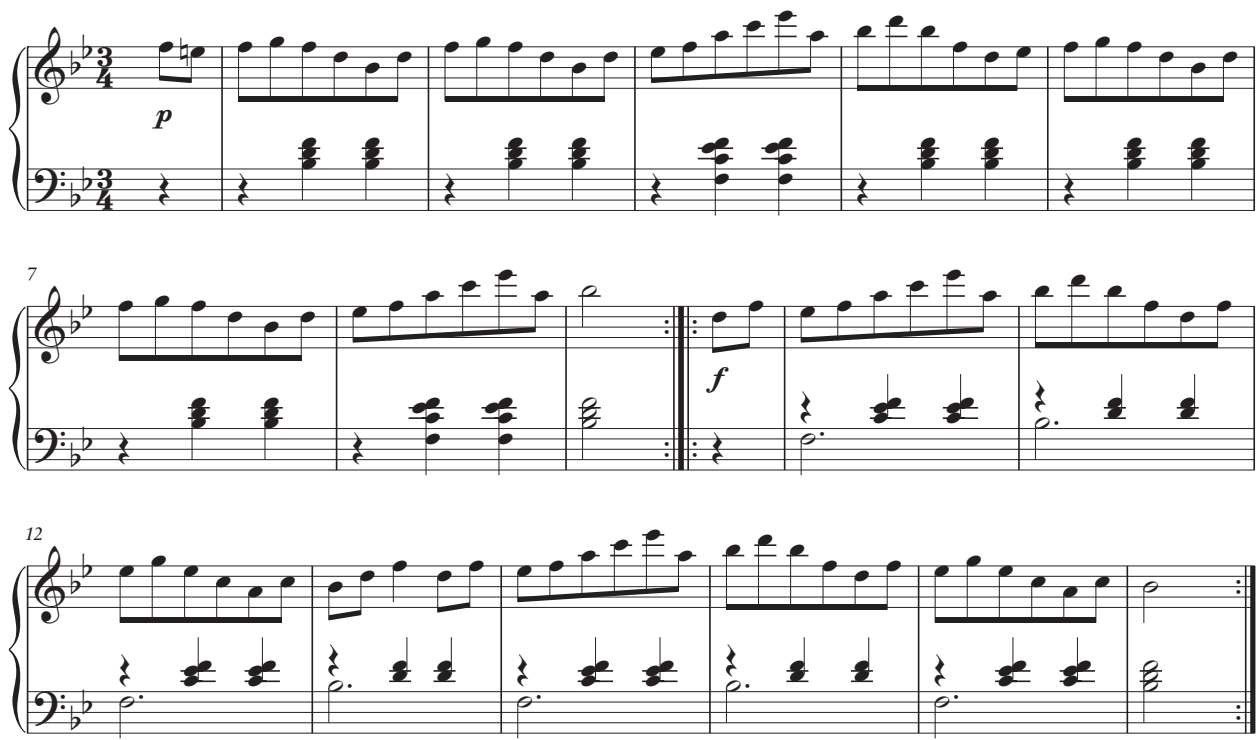

Beispiel 1: Ländlerische Formbildung: Franz Schubert, 8 Ländler, D 378 (1816), Nr. 2
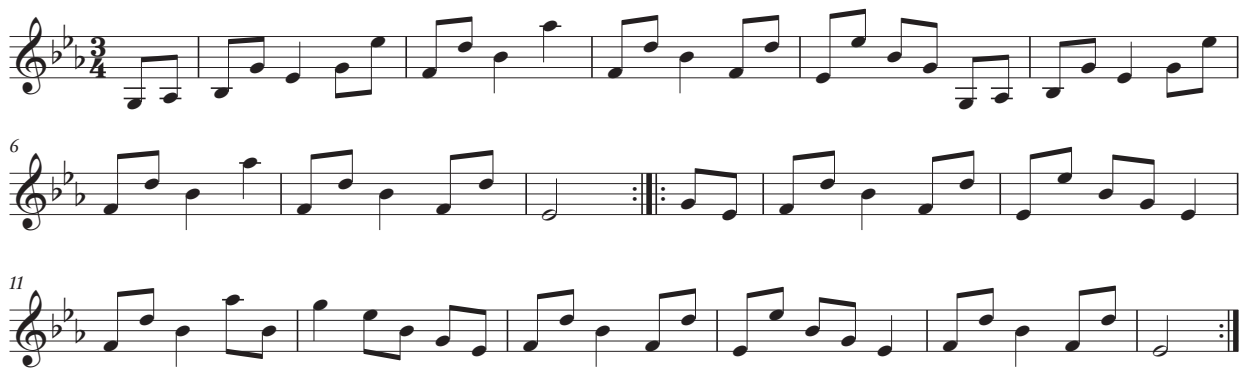

Beispiel 2: Dieselbe Formbildung nach einer Schrift aus der Volksmusikforschung ${ }^{11}$

Das formale Verfahren zur Bildung des B-Teils heißt in der Sprache der Ländlergeiger: »Der geht verkehrt." Gemeint ist insbesondere die Harmonik, da der B-Teil auf der Dominante statt auf der Tonika beginnt. ${ }^{12}$ Das `Verkehren` zählt zu den typisch alpenländisch-volkstümlichen Arten, eine zweiteilige Liedform zu bilden. Eine andere ist, den B-Teil als Variation zu setzen, wie hier bei Nummer 25 aus Schuberts D 365.

11 Derschmidt/Deutsch 1998, 70. Überliefert ist, wie üblich, nur die Melodiestimme. Die typische Ländlerbesetzung bestand aus zwei Violinen und Bass.

12 Vgl. ebd., 69 und 55. 

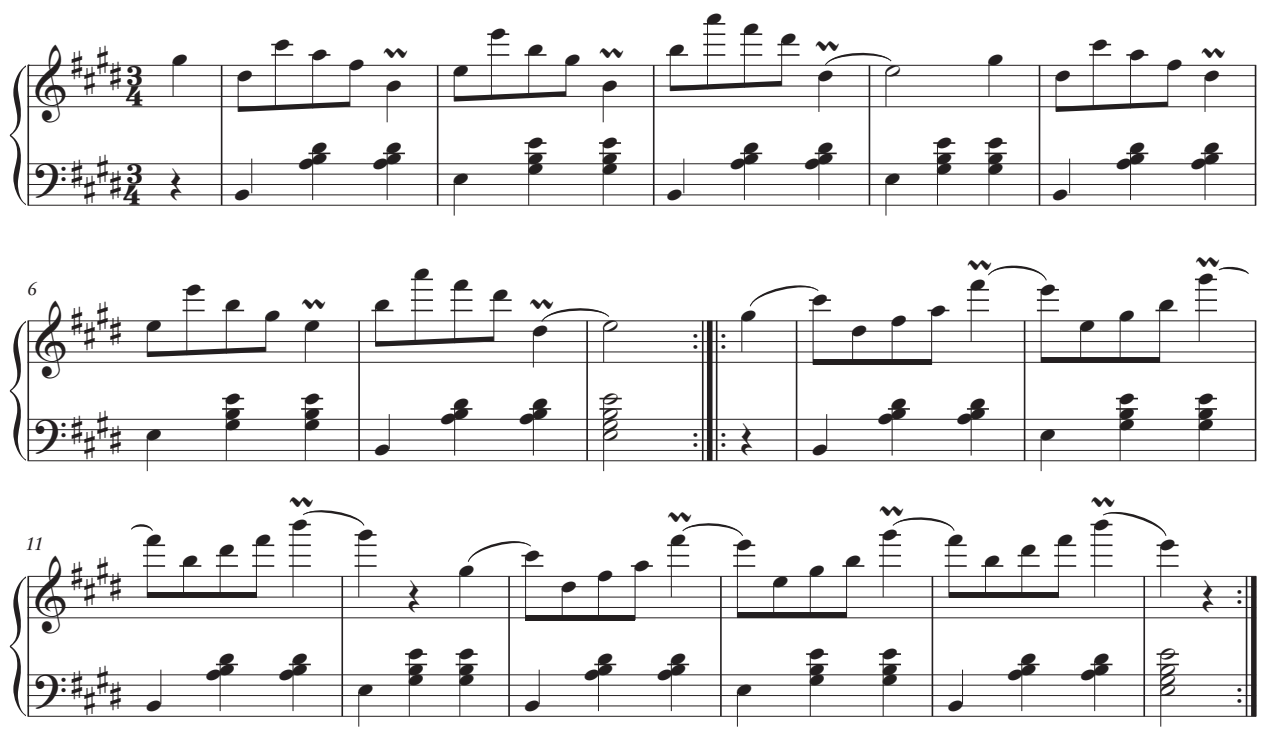

Beispiel 3: Franz Schubert, 36 Originaltänze für Klavier ${ }^{13}$, D 365 (erschienen 1821 als op. 9), Nr. 25

\section{Traditionell-kunstmusikalische Formbildung: Menuett}

Folgende Merkmale sind typisch für das Menuett der 2. Hälfte des 18. Jahrhunderts:

- Das Menuett ist 16, 20 oder 24 Takte lang.

- Das Menuett und seine Wiederholung rahmen ein Trio. Das Trio ist ebenfalls 16, 20 oder 24 Takte lang, und steht entweder in der gleichen oder der Varianttonart, häufiger aber (bei Menuetten in Dur) in der Dominant- oder Subdominanttonart.

- Das Menuett setzt sich aus mindestens zwei 8-Taktern zusammen. Soll nicht dazu getanzt werden, können sowohl die 8-Takter erweitert als auch ungeradzahlige Taktgruppen gebildet werden. Das typische Tanz- und Schulmenuett besteht jedoch aus Viertaktgruppen. Unabhängig von der Gesamtzahl der Takte ist das Menuett durch ein Wiederholungszeichen in zwei sReprisen geteilt.

- Der erste 8-Takter ist als Periode, Satz oder hybrider Typus gestaltet. Im Falle einer periodenartigen Gestaltung endet der eröffnende Vierer in der Regel halbschlüssig.

Eine weit verbreitete Formstrategie ist das reprisenartige Wiederaufgreifen des ersten Vierers durch den letzten. Der Formteil zwischen dem ersten Achttakter und der Reprise unterscheidet sich von den ihn flankierenden Teilen in der Regel durch stehende, pendelnde oder sequenzierende harmonische Strukturen. Auf diese Weise ergibt sich eine

13 Dieses später auch als 36 Erste Walzer gedruckte Werk ist offensichtlich eine Zusammenstellung von

Tänzen, die in ganz unterschiedlichen biographischen Kontexten entstanden sind. 
formfunktionale Differenzierung der Teile, die typisch für eine kunstmusikalische Stilistik ist. In der modernen Formenlehre wird der entsprechende Formtypus häufig als ıdreiteilige Liedform bezeichnet. ${ }^{14}$ In der zeitgenössischen Theorie des 18. Jahrhunderts, etwa bei Heinrich Christoph Koch oder Joseph Riepel, wird demgegenüber die übergeordnete Zweiteiligkeit der Form akzentuiert ${ }^{15}$, wobei die Wiederkehr des ersten Gedankens am Ende gleichberechtigt neben anderen Möglichkeiten steht, musikalischen Zusammenhang zu gewährleisten. Gleichwohl ist eine durch Reprisenmomente gestützte Dreiteiligkeit für die Menuette der Wiener Klassik so repräsentativ, daß es legitim erscheint, sie der volkstümlich-ländlerischen Formbildung idealtypisch gegenüberzustellen. ${ }^{16}$

- Beim Menuett wie bei den meisten anderen musikalischen Formen der Hochkultur (Suitensatz, Sonatensatz) steht am ersten Doppelstrich typischerweise die Oberquinttonart (in Moll auch die Paralleltonart).

Als Beispiel soll hier das 1816 entstandene Menuett Nr. 1 aus den 2 Menuetten D 380 dienen (Beispiel 4). Eher schwach ausgeprägt ist hier der Kontrast des Mittelteils, der hauptsächlich in der Dynamik besteht. Umso deutlicher ist die Wiederaufnahme der Takte 5-8 in den Takten 13-16.

Schubert überträgt diese eher kunstmusikalische Formbildung auch auf seine ländlerartigen Tänze, wenn auch selten so konsequent wie im folgenden Beispiel aus den 12 Deutschen Tänzen D 420 (Beispiel 5). Man kann an dieser Formbildung erkennen, dass es sich hier um einen Deutschen Tanz im engeren Sinne, also einen musikalisch mit dem Menuett eng verwandten Tanz handelt.

\section{Schwebender Soziolekt}

Aus dem Aufeinandertreffen und der Verbindung unterschiedlicher musikalischer Elemente, die ein bestimmtes soziologisches Assoziationspotential tragen, entsteht eine neue, individuelle Aussage. Dieser Prozess der musikalischen Semiose ist in der nordamerikanischen Musiktheorie der jüngeren Zeit ${ }^{17}$ genauer beschrieben worden. In diesem

14 William Caplin geht davon aus, dass die Mehrzahl der klassischen Rahmen-Menuette der "small ternary«-Form folgt. Durch die Wiederholungen der beiden Achttakter des Menuetts aber erscheine diese »small ternary«-Form in einer »rounded binary version« (1998, 220). Beide Aspekte - Zweiund Dreiteiligkeit - stehen für Caplin mehr oder weniger gleichberechtigt nebeneinander: »The small ternary and rounded binary must be understood, nonetheless, as essentially the same form; that is, they both contain the three functions of exposition, contrasting middle, and recapitulation « (ebd., 71).

15 Vgl. Budday 1983, 18, sowie Krämer 2012, 26 ff.

16 Freilich gehört das Menuett nicht per se der musikalischen Hochkultur an, sondern kann niedere wie höhere Stilebenen ausprägen, die sich auf der Ebene der Form in einem weniger oder mehr von Subordination der Taktgruppen zeigt. So ist eine deutlich ausgeprägte Dreiteiligkeit unter Mozarts zahlreichen Menuetten für Festivitäten eher selten, gehört aber zum Standard (selbst der Trios) der Instrumentalzyklen von Solosonate, Kammermusik und Sinfonie.

17 Vgl. Hatten 1994 und 2004. 

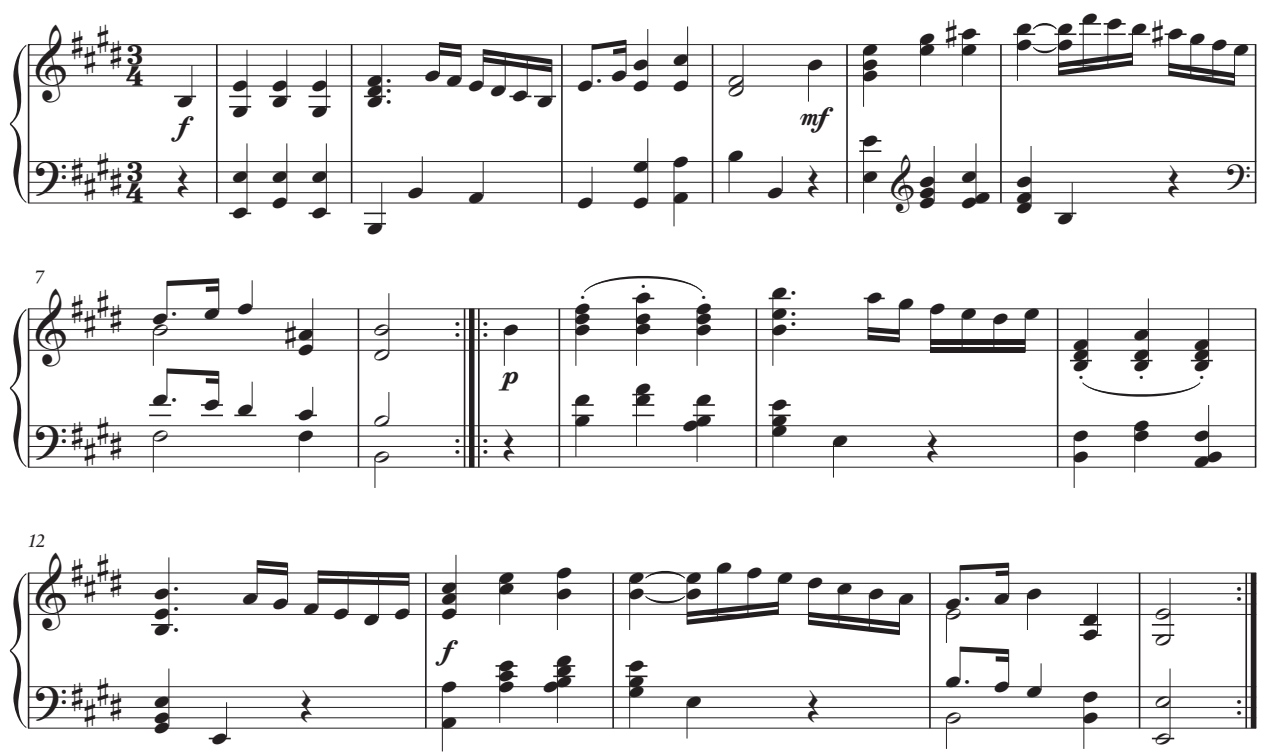

Beispiel 4: Franz Schubert, 3 Menuette (Nr. 3 Fragment), D 380 (1816), Nr. 1
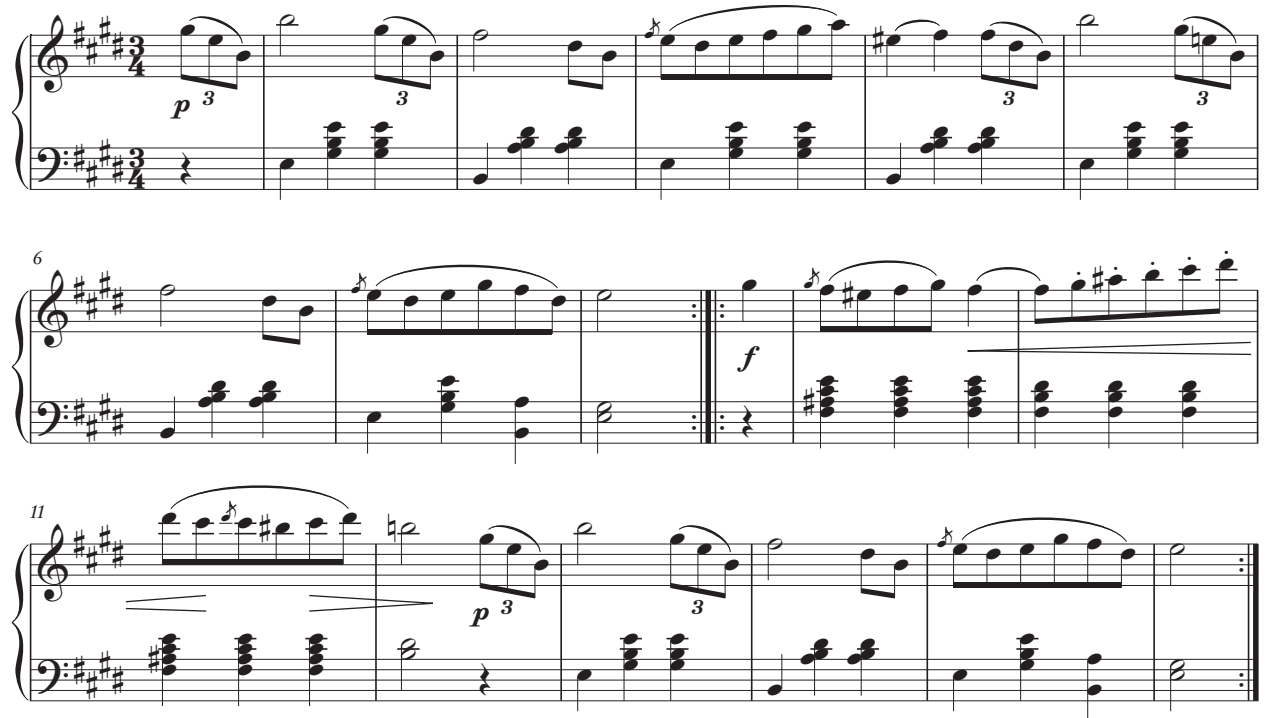

Beispiel 5: Franz Schubert, 12 Deutsche Tänze, D 420 (1816), Nr. 7 
Sinne gelten musikalische Elemente und Gestaltungsweisen als >markiert $(» \text { marked «) })^{18}$, wenn sie sich von einem sunmarkierten` Umfeld als Träger bestimmter Bedeutungen abheben. ${ }^{19}$

Schuberts ischwebender Soziolektı resultiert demnach aus der Neukombination und Bezugsetzung markierter musikalischer Soziolekte. Eine Analyse der Semantik müsste im Einzelnen auseinandersetzen, welche Konnotationen sich zu Schuberts Zeit mit den von ihm aufgegriffenen Stilen verbanden, und eine Deutung der neu entstehenden Zusammenhänge vornehmen. Die zentrale Zielsetzung des vorliegenden Aufsatzes ist es demgegenüber, auf einer technischen Ebene deutlich zu machen, wie mit Hilfe der musikalischen Form verweisende Strukturen zur Wahrnehmung gelangen und miteinander kombiniert werden können.

Aus welchem Anlass Schuberts Tänze entstanden sind und in welchem rezeptionellen Zusammenhang sie stehen, ist aus den gedruckten Sammlungen nicht immer ersichtlich: Stücke, die wie Tanzmusik-Improvisationen wirken, stehen neben Albumblättern und Widmungskompositionen. Sämtliche Zwischenstufen vom unangefochtenen Ländler bis hin zum lyrisch stilisierten Tanzsatz sind vertreten. Das Extrem auf der volksmusikalischen Seite wurde bereits mit Beispiel 1 demonstriert. In den allermeisten Schubert-Tänzen werden jedoch volksmusikalische Stilmittel mit lyrischen Gestaltungsmitteln kombiniert. Um das andere, slyrische` Ende der Skala zu erkunden, eignen sich besonders gut die oben erwähnten Widmungskompositionen. Zu letzterem Typus tendieren unter anderem die von Schubert für den Klavierunterricht der Prinzessinnen Esterházy in Szelisz verfassten Stücke.

\section{Vermischung von lyrischer und ländlerischer Gestaltung}

\section{Ländlerischer B-Teil aus lyrischem A-Teil}

Ein häufiges Verfahren bei Schubert besteht darin, den A-Teil dem Typ des lyrischen Klavierstücks anzunähern, den B-Teil jedoch in einer für den Ländler charakteristischen Weise davon abzuleiten (Beispiel 6).

Der hier wiedergegebene Ländler erweist sich schon auf den ersten Blick durch die Tonartenwahl und die Vorschrift dolce als dem Bereich des privat-intellektuellen Musiklebens zugehörig. In dieselbe Richtung weist auch der Umstand, dass in den Takten 2 und 3 an die Stelle der erwarteten Tonika-Dominant-Beziehung ein Farbwechsel zwischen Tonika und Tonikaparallele tritt. Formal steht der erste Achttakter dem Thementypus `Satzı nahe, der gegenüber der ländlertypischen Periode eine höhere formale Komplexität aufweist.

Der zweite Achttakter schlägt demgegenüber deutlich in die Ländler-Richtung aus. Das Verfahren, den B-Teil aus der Schlussformel des A-Teils zu generieren (`der geht

18 Vgl. Hatten 2004, 34 ff.

19 Der Einsatz markierter Strukturen in einem neuen Zusammenhang bedeutet eine Bearbeitung, im englischen trope genannt, durch die neue Bedeutungen entstehen. Das Konzept der tropes stammt aus der Literaturwissenschaft. Vgl. dazu ebd., 68, sowie Bloom 1997. 

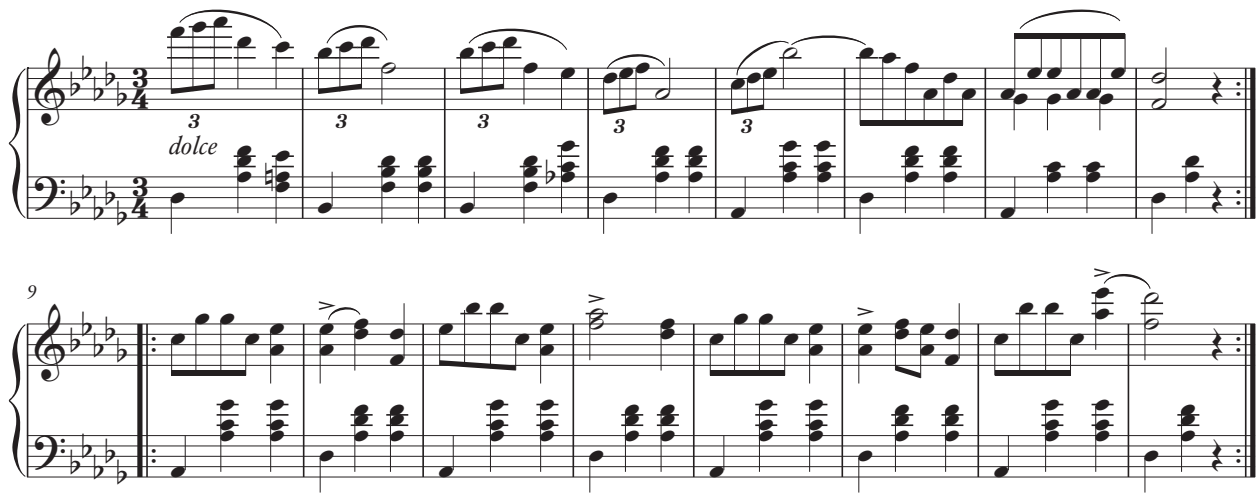

Beispiel 6: Franz Schubert, Zwölf Walzer, siebzehn Ländler und neun Ecossaisen op. 18, D 145 (erschienen 1823), Ländler Nr. 5

verkehrt`), ist typisch für die Volksmusik. Dem entsprechen das konsequente T-D-Pendel und der einfache Periodenbau mit imperfektem Ganzschluss in der Mitte und perfektem Ganzschluss am Ende.

\section{Ländler-Form, lyrische Gestaltung}

Das folgende Stück entstammt derselben Sammlung. Es entstand in Schuberts Zeit als Klavierlehrer im Hause Esterházy. ${ }^{20}$ Die Achtelketten der Melodiestimme scheinen die Valse brillante Chopins vorwegzunehmen.
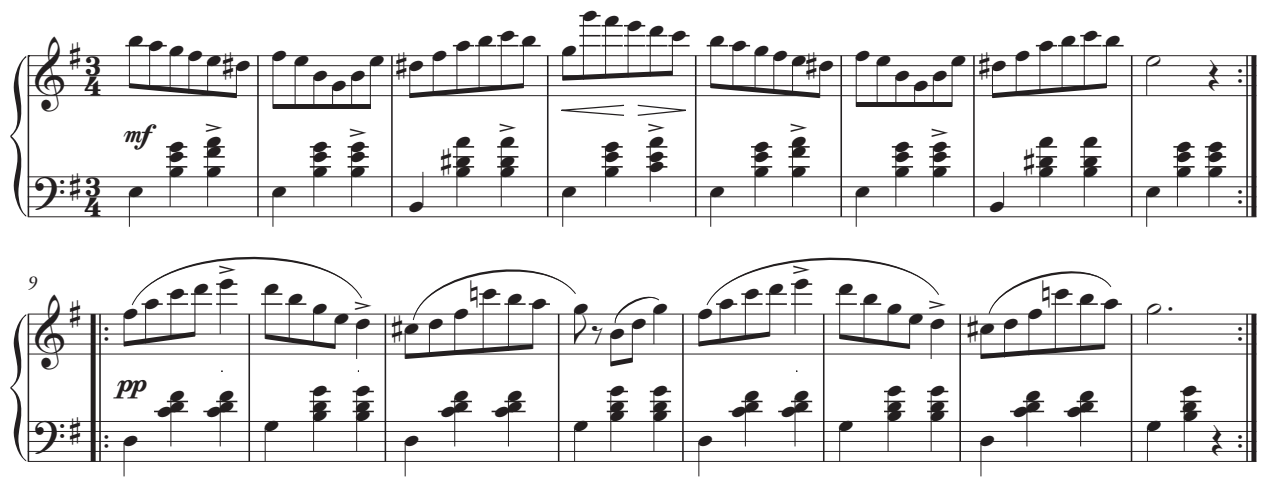

Beispiel 7: Franz Schubert, Zwölf Walzer, siebzehn Ländler und neun Ecossaisen op. 18, D 145 (erschienen 1823), Walzer Nr. 5

20 In den drei Manuskripten Schuberts, die das Stück enthalten, ist es jeweils als `Deutscher überschrieben und steht in es-Moll, einer Tonart, die große Distanz zum einfachen Ländler-Stil signalisiert. Die Tonart wurde in der Erstveröffentlichung vermutlich von Schubert selbst im Interesse einer geschlosseneren Tonartenfolge ( $\mathrm{E}, \mathrm{H}, \mathrm{a}, \mathrm{cis}, \mathrm{e}, \mathrm{h}, \mathrm{Es}$, es, fis, h, H, E) nach e-Moll verändert. 
In formaler Hinsicht unterscheidet sich dieses Stück nicht von den einfachsten Ländlern: Der A-Teil ist als Periode mit imperfektem und perfektem Ganzschluss gebildet. Der B-Teil ergänzt ihn zu einer zweiteiligen Liedform ohne Reprisenereignis. Er steht komplett in der parallelen Durtonart; eine Rückmodulation erfolgt nicht. Für Kompositionen des frühen 19. Jahrhunderts ist diese Verweigerung der elementarsten Art formaler Geschlossenheit - der Rückkehr zur Grundtonart - sehr ungewöhnlich.

\section{Lyrische Gestaltung, Reste ländlerischer Formbildung}

Die folgende Widmungskomposition aus dem Album der Anna (`Netty`) Hönig (verheiratete Mayerhofer von Grünbühel) trägt in Schuberts Handschrift keinen Titel, wurde aber bei ihrer Erstveröffentlichung in der Alten Gesamtausgabe 1897 mit /Walzer überschrieben. Das Stück weist hinsichtlich Begleitung und Motivik überhaupt keine Merkmale des typischen Ländlerstils mehr auf und stellt insofern einen Extrempunkt unter Schuberts Tanzstücken dar.
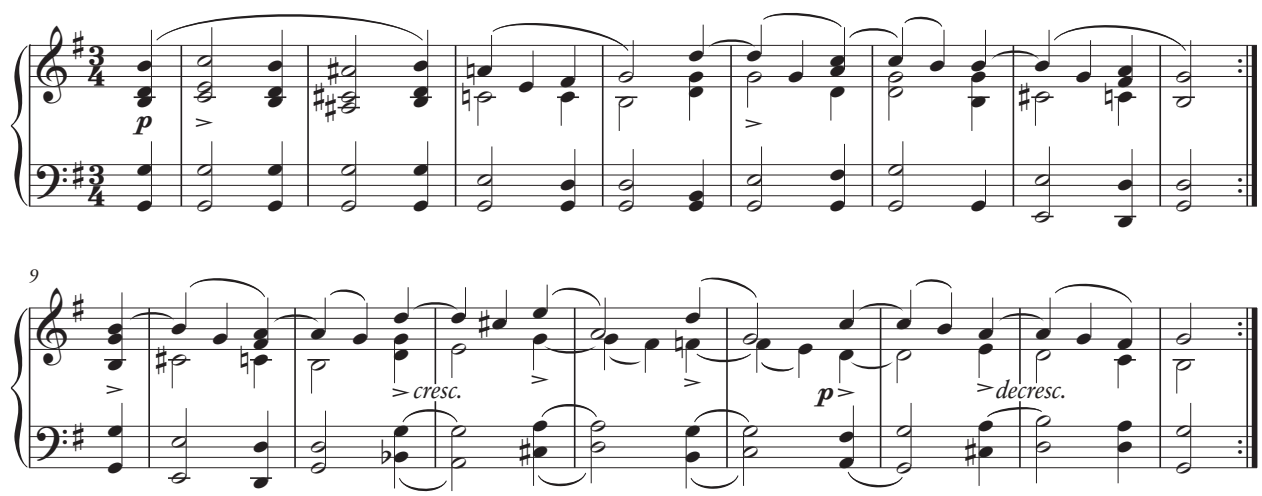

Beispiel 8: Franz Schubert, "Walzer in G», D 844 (1825)

In formaler Hinsicht jedoch erweist sich die Komposition als Schuberts Tanzstücken zugehörig. Erstaunlich ist beispielsweise die Nähe zur Formbildung des vorhin besprochenen Ländlers aus D 145 (Beispiel 7):21 Das seltsam unhierarchische zweimalige Schließen des ersten Achttakters auf der ersten Stufe erinnert trotz der ansonsten eher satzartigen Formulierung an die Ländlerperiode. Die Motivik des zweiten Achttakters speist sich aus der Schlussformel des ersten, wie wir es ganz ähnlich auch im Ländler Nr. 5 aus D 145 (Beispiel 6) und im Ländler Nr. 2 aus D 378 (Beispiel 1) gesehen haben. Des Weiteren changiert der Satz wie auch zahlreiche andere Tanzsätze Schuberts zwischen zwei- und dreiteiliger Form.

Obgleich in den Takten 9-12 keine regelrechte Sequenzierung stattfindet, ähnelt die Passage durch die wiederholten Vorhaltsbildungen der Ober- bzw. Mittelstimme

21 Dafür, dass es sich um ein Tanzstück handelt, spricht auch die auffällige Nähe zu den Trios der Scherzos aus den Sonaten D 840, 845 und 850, die Schubert zur selben Zeit konzipierte. 
(T. 9-10, 10-11 und 11-12) einem sequenzierenden >Mittelteilı. Auch das Erreichen der V. Stufe in Takt 12 ist ein Mittelteil-Merkmal. Allerdings schließen die Takte 13-16 nicht, wie es in einer >dreiteiligen Liedform zu erwarten wäre, auf den ersten Gedanken zurück, sondern auf den zweiten Vierer, also die Takte 5-8. Dabei handelt es sich um eine auch in den Menuetten der Wiener Klassik übliche Formbildung.

Für die generelle Zweiteiligkeit dieses Stückes spricht darüber hinaus, dass die Zäsur in Takt 12, die durch die schwache Kadenzierung nach D-Dur ohnehin bloß angedeutet ist, überspielt wird: Ausgerechnet die Takte 12 und 13 werden durch melodische und harmonische Sequenzierung (A-D, G-C) zusammengefasst. Die zweiteilige Liedform spricht ebenso wie die fehlende Dominant-Tonika-Hierarchie der Schlüsse im A-Teil für eine Verwandtschaft mit länderartigen 16-Taktern. Die Form des Satzes ist im Wesentlichen beiordnend und undramatisch. In ihr verbinden sich ländlerische Formbildung und lyrischer Stil.

Die historische Entwicklungslinie der Tanzmusik verläuft von den städtischen LändlerAdaptionen zur großen Walzer-Kette von Johann Strauß und Sohn. Merkmale des arrivierten Walzers sind die formale Vereinheitlichung der zunächst nur reihenden Walzer-Sammlung durch Introduktion und Coda sowie die längeren Melodiebögen von mindestens 16 Takten.

Bei Schubert nehmen die ländlerartigen Tänze einen anderen Weg hin zum lyrischen Klavierstück. ${ }^{22}$ Die Periodik bleibt achttaktig; rahmende Teile finden sich nicht. Vielmehr tendieren Schuberts musikalische Miniaturen vielfach zu einem zurückgenommenen, privaten Gestus und einer avancierten harmonische Gestaltung. Frappierend ist das lückenlose Spektrum aller Grade von Einfachheit und Komplexität, das uns im Schubertschen Tanzrepertoire begegnet. Besonders interessant sind Stücke, deren Soziolekt zwischen der ländlerischen und der lyrischen Sphäre schwebt. Oft bestimmen dezidierte Volksmusik-Techniken die Formbildung in ansonsten der kunstmusikalischen Sphäre angehörigen Stücke. Hier lässt sich im Kleinen jene Verbindung Schuberts zur süddeutschen und alpenländischen Folklore nachvollziehen, die in einer schwerer bestimmbaren Weise auch seine größeren Werken prägt.

\section{Noten}

Schubert, Franz (1990): „Tänze«, in: Neue Ausgabe sämtlicher Werke, Serie VII: Klaviermusik. Abteilung 2: Werke für Klavier zu zwei Händen, Bd. 7, hg. von der Internationalen Schubert-Gesellschaft, vorgelegt von Walburga Litschauer, Kassel u.a.: Bärenreiter.

22 Mit der Bedeutung von Schuberts Tänzen für Schumanns Klavierminiaturen beschäftigt sich sehr detailliert die Dissertation von Marie Luise Maintz (1995). Zu »Schuberts Tänze[n] als romantische Musik« siehe Mahlert 2005, 179-186. 


\section{Literatur}

Bloom, Harold (1997), The Anxiety of Influence: A Theory of Poetry, Oxford: Oxford University Press.

Budday, Wolfgang (1983), Grundlagen musikalischer Formen der Wiener Klassik, Kassel u. a.: Bärenreiter.

Caplin, William E. (1998), Classical Form, Oxford: Oxford University Press.

Deutsch, Walter / Annemarie Gschwantler (1994), "Steyerische Tänze«, in: Corpus Musicae Popularis Austriacae, Bd. 2: Steiermark, hg. vom Steirischen Volksliedwerk, Wien: Böhlau.

Derschmidt, Volker / Walter Deutsch (1998), »Der Landler«, in: Corpus Musicae Popularis Austriacae, Bd. 8: Volksmusik in Oberösterreich, hg. vom Oberösterreichischen Volksliedwerk, Wien: Böhlau.

Gmeiner, Josef (1979), Menuett und Scherzo, Tutzing: Schneider.

Hatten, Robert (1994), Musical Meaning in Beethoven, Bloomington, Indianapolis: Indiana University Press.

(2004), Interpreting Musical Gestures, Topics, and Tropes - Mozart, Beethoven, Schubert, Bloomington, Indianapolis: Indiana University Press.

Hilmar, Ernst (1985), Franz Schubert in seiner Zeit, Wien: Böhlau.

Karbusicky, Vladimir (1986), Grundriß der musikalischen Semantik, Darmstadt: Wissenschaftliche Buchgesellschaft.

Krämer, Laura (2012), »Formstrategien der Zweiteiligkeit in Mozarts Menuetten«, Musik \& Ästhetik 63, 7/2012, 25-42.

Mahlert, Ulrich (2005), »Bodenhaftung und Transzendenz. Zu Schuberts Tänzen«, in: Zwischen Komposition und Hermeneutik. Festschrift für Hartmut Fladt, hg. von Ariane Jeßulat, Andreas Ickstadt und Martin Ullrich, Würzburg: Könsigshaus \& Neumann, 168-186.

Maintz, Marie Luise (1995), Franz Schubert in der Rezeption Robert Schumanns, Kassel u. a.: Bärenreiter. 